\title{
Transverse decoherence and coherent spectra in long bunches with space charge
}

\author{
Vladimir Kornilov \\ GSI Helmholtzzentrum für Schwerionenforschung, Planckstraße 1, 64291 Darmstadt, Germany \\ Oliver Boine-Frankenheim \\ GSI Helmholtzzentrum für Schwerionenforschung, Planckstraße 1, 64291 Darmstadt, Germany \\ Technische Universität Darmstadt, Schlossgartenstraße 8, 64298 Darmstadt, Germany
}

(Received 7 May 2012; published 6 November 2012)

\begin{abstract}
The transverse bunch spectrum and the transverse decoherence/recoherence following an initial bunch offset are important phenomena in synchrotrons and storage rings, and are widely used for beam and lattice measurements. Incoherent shifts of the particles betatron frequency and of the synchrotron frequency modify the transverse spectrum and the bunch decoherence. In this study we analyze the effects of transverse space charge and of the rf nonlinearity on the decoherence signals. The transverse bunch decoherence and the resulting coherent spectra are measured in the SIS18 synchrotron at GSI Darmstadt for different bunch parameters. The frequencies of the bunch head-tail modes provide a direct measure for the self-field space charge tune shift. Particle tracking simulations together with an analytical model are used to describe the modifications in the decoherence signals and in the coherent spectra due to space charge and the rf bucket nonlinearity.
\end{abstract}

DOI: 10.1103/PhysRevSTAB.15.114201

PACS numbers: 29.27.- a, 29.20.dk

\section{INTRODUCTION}

Transverse coherent oscillations of bunches induced by a fast kicker magnet are routinely used in synchrotrons or storage rings to measure, for example, the tune or other ring parameters, see e.g. [1]. The transverse offset of a bunch, averaged over the bunch length, can be recorded every single turn. The spectrum is then concentrated around the base-band $Q_{f 0} f_{0}$, where $Q_{f 0}$ is the fractional part of the betatron tune $Q_{0}$ and $f_{0}$ is the revolution frequency. This diagnostics is usually used for timeresolved and very accurate measurements of the tune $Q_{f 0}$.

Transverse bunch decoherence is a process of a turn-toturn reduction of the total bunch offset signal after an initial bunch displacement. In a linear focusing lattice the bunch decoherence is a manifestation of the lattice chromaticity $\xi$ where the synchrotron dynamics also plays an important role, causing the signal recoherence exactly after the synchrotron period. Other damping mechanisms, as due to lattice nonlinearities, additionally damp the transverse oscillations. Transverse decoherence is often used as a machine diagnostics tool. Undesired transverse bunch oscillations can also appear after the bunch-to-bucket transfer between synchrotrons. In order to use transverse decoherence as a diagnostics tool for intense bunches of arbitrary length and also to control undesired oscillations of such bunches, it is important to understand the

Published by the American Physical Society under the terms of the Creative Commons Attribution 3.0 License. Further distribution of this work must maintain attribution to the author(s) and the published article's title, journal citation, and DOI. decoherence in the presence of transverse space charge and nonlinear synchrotron oscillations.

We demonstrate that the decoherence signal can be explained in terms of the transverse head-tail bunch mode spectrum. For finite chromaticity also the $k>0$ head-tail modes contribute to the bunch coherent spectrum. The shift of the head-tail mode frequencies due to space charge and wall currents can be well explained in terms of the analytical expressions for an airbag bunch distribution $[2,3]$. The head-tail mode frequencies are also modified by changes in the individual particle synchrotron frequency. In long bunches, one has to account for the spread of the synchrotron frequencies. Both transverse space charge and nonlinear synchrotron oscillations are important to understand the decoherence signals and transverse spectra. We demonstrate that, once the spectrum and decoherence modifications are understood, they can be used to extract useful information about the bunches.

In this paper we describe measurements of transverse bunch spectra and decoherence signals obtained in the heavy-ion synchrotron SIS18 at GSI Darmstadt. The observed modification of the head-tail spectrum and of the decoherence signal caused by transverse space charge and nonlinear synchrotron oscillations are explained in terms of our theoretical approach. This approach is based on an expansion of an analytical theory for head-tail modes in combination with particle tracking simulations.

In Sec. II we use theoretical and numerical approaches to analyze the effects of space charge and nonlinear synchrotron motion on the transverse spectra and on the bunch decoherence signal. We show that a simple model for the head-tail mode frequencies with fitting parameters can be 
used to explain the numerically obtained spectrum modifications as well as the bunch decoherence as a function of the chromaticity. In Sec. III the results of measurements performed at the SIS18 synchrotron are presented. The space charge tune shifts determined from the transverse spectra are summarized, the role of nonlinear synchrotron motion is demonstrated and transverse bunch decoherence signals measured for different bunch conditions are presented and explained. The work is concluded in Sec. IV.

\section{THEORY AND NUMERICAL SIMULATIONS}

The Fourier transformation of the transverse bunch signal provides peaks at frequencies which represent the bunch eigenmodes, also called head-tail modes. For short, low-intensity bunches (the synchrotron frequency $f_{s}=$ $Q_{s} f_{0}$ does not depend on the amplitude, no collective effects), the transverse spectrum has peaks at $\Delta Q=Q-$ $Q_{f 0}=0$ for $k=0, \Delta Q= \pm Q_{s}$ for $k= \pm 1, \Delta Q= \pm 2 Q_{s}$ for $k= \pm 2$, and so forth. Collective effects, like transverse space charge or ring impedances, change the bunch eigenfrequencies and thus shift the peaks in the transverse spectrum.

Transverse space charge effects are described by the characteristic tune shift,

$$
\Delta Q_{\mathrm{sc}}=\frac{\lambda_{0} r_{p} R}{\gamma^{3} \beta^{2} \varepsilon_{\perp}},
$$

where $R$ is the ring radius, $\beta$ and $\gamma$ are the relativistic parameters, $r_{p}=q_{\mathrm{ion}}^{2} /\left(4 \pi \epsilon_{0} m c^{2}\right)$ is the classical particle radius, $\lambda_{0}$ is the peak line density (at the bunch center), and $\varepsilon_{\perp}$ is the transverse total emittance. This tune shift corresponds to a round cross section with a transverse $\mathrm{K}-\mathrm{V}$ distribution, with the dispersion zero, and is defined as the modulus of the negative shift. In a rms-equivalent bunch with the Gaussian transverse profile, i.e., the transverse rms emittance is $\varepsilon_{x}=\varepsilon_{\perp} / 4$, the maximum space charge tune shift is twice this value, $\Delta Q_{\mathrm{sc}}^{\max }=2 \Delta Q_{\mathrm{sc}}$. In the case of an elliptic transverse cross section with the rms emittances $\varepsilon_{y}, \varepsilon_{x}$, the parameter $\varepsilon_{\perp}$ in Eq. (1) should be replaced by

$$
\varepsilon_{\perp}=2\left(\varepsilon_{y}+\sqrt{\varepsilon_{y} \varepsilon_{x} \frac{Q_{0 y}}{Q_{0 x}}}\right),
$$

here for the vertical $(y)$ plane, for the horizontal plane correspondingly. The tune ratio $Q_{0 y} / Q_{0 x}$ stems from the average along the ring of the beta function ratio, variations of which should be taken into account for a more accurate tune shift calculation for a specific lattice. The parameter for the effect of space charge in a bunch is defined as a ratio of the characteristic space charge tune shift Eq. (1) to the small-amplitude synchrotron tune,

$$
q=\frac{\Delta Q_{\mathrm{sc}}}{Q_{s 0}}
$$

\section{A. Longitudinal dipole frequency}

An important parameter for head-tail bunch oscillations in long bunches [4-6] is the effective synchrotron frequency which will be different from the small-amplitude synchrotron frequency in short bunches. We will show that in long bunches the longitudinal dipole frequency $Q_{\text {dip }} f_{0}$ can be chosen as a substitute for the small-amplitude incoherent synchrotron frequency $Q_{s 0} f_{0}$. The longitudinal coherent dipole frequency can be accurately measured from the bunch signal, as we will show in the experimental part of this paper. It should be however mentioned that other choices for the characteristic synchrotron frequency in long bunches are possible and can be useful depending on the consideration contents. The frequency of smallamplitude dipole oscillations can be calculated as $[4,6]$

$$
\frac{Q_{\mathrm{dip}}^{2}}{Q_{s 0}^{2}}=\frac{2 R}{N_{p} h} \int_{0}^{\tau_{\max }} \frac{V_{\mathrm{rf}}}{V_{0}} \lambda^{\prime}(\tau) d \tau,
$$

where for the single rf the voltage form is $V_{\mathrm{rf}}=V_{0} \sin (\tau)$, $h$ is the rf harmonic number, $N_{p}$ is the particle number, $\tau=$ $z h / R$ is $\mathrm{rf}$ bucket radian, and $\lambda$ is the line density. The small-amplitude bare synchrotron tune is given by

$$
Q_{s 0}^{2}=\frac{q_{\text {ion }} V_{0} h|\eta|}{2 \pi m \gamma \beta^{2} c^{2}}
$$

where $\eta$ is the machine slip factor. The dependence of $Q_{\text {dip }}$ on the rms bunch length $\sigma_{z}$ for a Gaussian bunch is shown (red curve) in Fig. 1. The bunch length $\sigma_{z}$ is dimensioned in radians of the rf bucket, i.e. $\sigma_{z}=L_{\mathrm{rms}} h / R$, where $L_{\mathrm{rms}}$ is the rms bunch length in meter.

In a realistic bunch in experiment, the bunch profile is normally different from an analytic model, thus we consider here another bunch form for comparison. For a parabolic longitudinal distribution (or elliptic bunch) with the total half-length $\tau_{p}=\sqrt{5} \sigma_{z}$ one obtains the analytic expression [6],

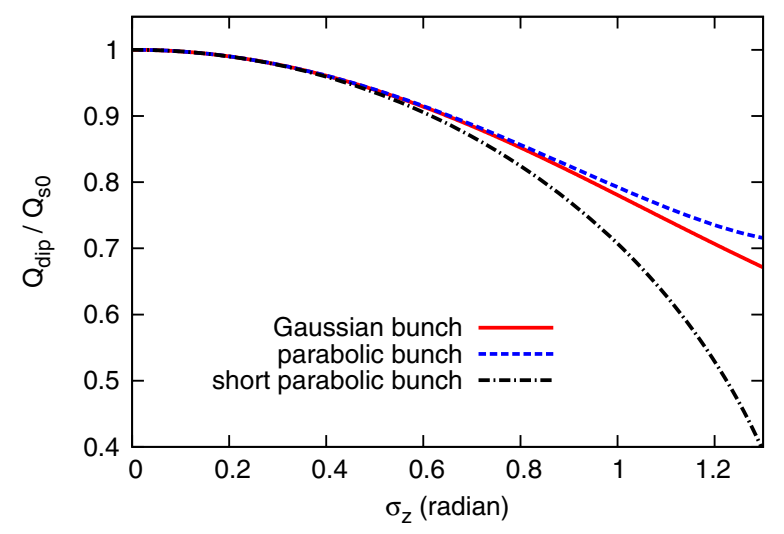

FIG. 1. The longitudinal dipole oscillation frequency as a function of the rms bunch length. The red curve is obtained using Eq. (4) for a Gaussian bunch, the blue dashed curve is given by Eq. (6), and the back chain curve is given by Eq. (7). 


$$
\frac{Q_{\mathrm{dip}}^{2}}{Q_{s 0}^{2}}=\frac{2 \tau_{p}-\sin \left(2 \tau_{p}\right)}{4 \sin \left(\tau_{p}\right)-4 \tau_{p} \cos \left(\tau_{p}\right)},
$$

which can be approximated in the case of a short bunch as

$$
\frac{Q_{\mathrm{dip}}}{Q_{s 0}}=\sqrt{1-\frac{\sigma_{z}^{2}}{2}} .
$$

From the comparison in Fig. 1, it follows that for short bunches with $\sigma_{z} \lesssim 0.6 \mathrm{rad}$ the approximation Eq. (7) is sufficient. For long bunches with $\sigma_{z} \gtrsim 1 \mathrm{rad}$ the dipole frequencies for Gaussian and parabolic bunches start to differ.

\section{B. Spectrum of a long bunch with space charge}

We use particle tracking simulations $[7,8]$ in order to investigate the combined effect of space charge and nonlinear synchrotron motion on transverse head-tail oscillations. The numerical codes have been validated [9] using analytic results [2]. For the transverse space charge force, a frozen electric field model is used, i.e., a fixed potential configuration which follows the center of mass for each bunch slice. This approach is justified in the rigid-slice regime and can be considered as a reasonable approach for moderate and strong space charge $[10,11]$. The applicability for complicated processes as Landau damping, especially for the weak/moderate space charge, should be studied in further papers. A round transverse cross section and a Gaussian transverse beam profile were used in the simulations in this work.

Figure 2 demonstrates differences in the transverse mode frequencies for bunches of different lengths, and with all the other parameters kept identical, including the space charge parameter $q=8$. The three lowest-order

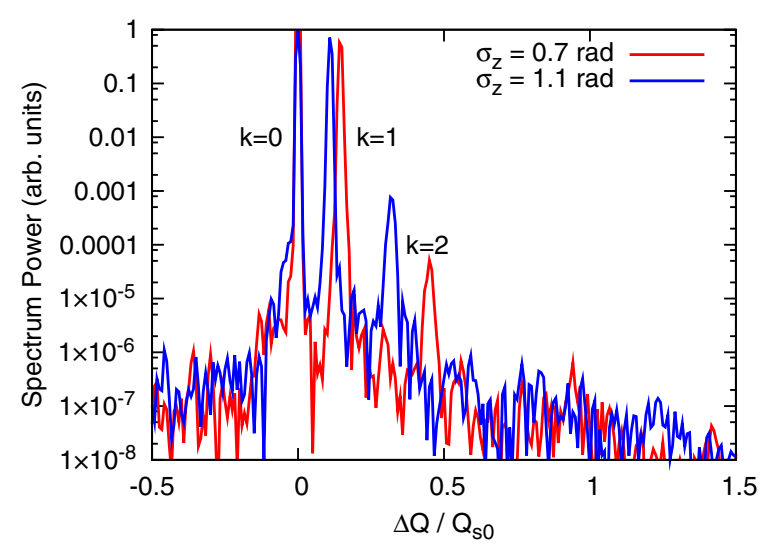

FIG. 2. Example transverse spectra of long bunches from particle tracking simulations, with space charge and nonlinear synchrotron motion taken into account. Bunches with two different rms length $\sigma_{z}$ are assumed, the space charge parameter $q=8$ and the bare synchrotron tune $Q_{s 0}$ is kept constant. The spectra clearly show the head-tail modes $k=0, k=1$, and $k=$ 2 . The tune shift $\Delta Q$ is related to the bare betatron tune as $\Delta Q=$ $Q-Q_{f 0}$. modes can be seen very well; the modes of the longer bunch are shifted closer to the bare betatron tune than those of the shorter bunch. In order to describe the bunch spectrum for arbitrary bunch length and space charge strength, simulation scans over different parameters have been performed. Our simulation results suggest that the airbag bunch model [2] can be applied to the head-tail modes in a long Gaussian bunch,

$$
\frac{\Delta Q_{k}}{Q_{s 0}}=-\frac{q}{2} \pm \sqrt{\frac{q^{2}}{4}+k^{2} q_{*}^{2}},
$$

where $q_{*}=Q_{s^{*}} / Q_{s 0}$ is a characteristic parameter depending on the bunch length and the nonlinear synchrotron oscillations. In our case $q_{*}$ is used as a fitting parameter. Keeping the space charge parameter constant, the bunch length has been varied and the resulting eigenfrequencies analyzed, see Fig. 3 for a scan with $q=8$. We observe substantial changes in the bunch mode frequencies with increasing bunch length. The parameter $q_{*}$ has been obtained from these simulation scans. Figure 4 shows a comparison between simulation results and the model Eq. (8) for a fixed bunch length and for different space charge parameters. The plot demonstrates that the model Eq. (8) is fairly accurate over the parameter range of the interest. As we additionally show in Fig. 4, there is a small difference between transverse Gaussian bunch profiles (with nonlinear transverse space charge) and transverse $\mathrm{K}-\mathrm{V}$ distributions (with linear space charge). In our simulations we use the more realistic Gaussian profile.

The chain curves in Fig. 3 show that it would be not correct to use the longitudinal dipole tune $Q_{\text {dip }}$ for the parameter $Q_{s^{*}}$. An interesting observation is that the type of the dependence of the mode frequencies on the bunch length is similar to $Q_{\text {dip }}$, being however slightly different. Also, the scale factor between $Q_{\text {dip }}$ and the real $\Delta Q$ is quite different for $k=1$ and $k=2$. The bare synchrotron tune,

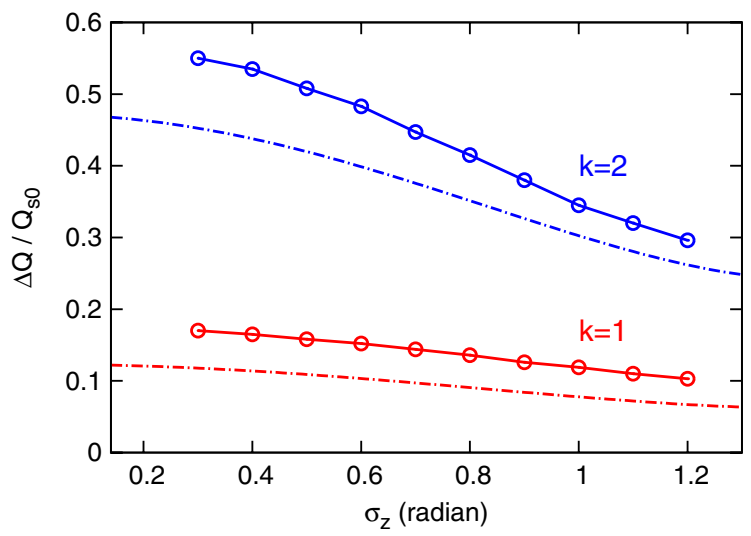

FIG. 3. Results of a simulation scan (circles) over the rms bunch length for a bunch with space charge parameter $q=8$. Red corresponds to the $k=1$ head-tail mode and blue to $k=2$ modes. For comparison, the chain curves show an estimation using Eq. (8) with $q_{*}=Q_{\text {dip }} / Q_{s 0}$. 


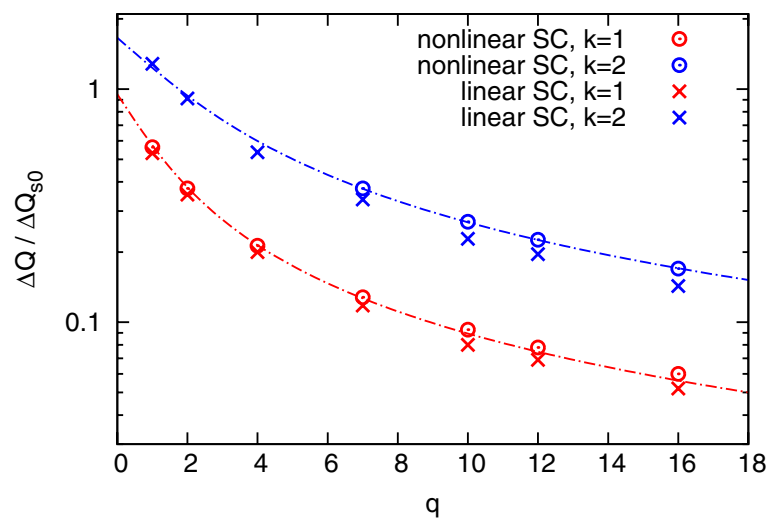

FIG. 4. Results of a simulation scan over the space charge parameter for a bunch with the rms length $\sigma_{z}=1.06 \mathrm{rad}$. The crosses show the eigenfrequencies of the modes $k=1$ and $k=2$ for bunches with a transverse K-V distribution, while the circles are for bunches with a Gaussian transverse profile. The chain curves are given by Eq. (8) with the coefficients $q_{*}=0.95$ for $k=1$ and $q_{*}=0.83$ for $k=2$, which corresponds to the results summarized in Fig. 5.

which would mean $q_{*}=1$, is not an adequate value, too, $\Delta Q$ would then be a constant for changing bunch length and it would correspond to the value of the chain curve at small $\sigma_{z}$.

Simulation results for practical usage are presented in Fig. 5. These $q_{*}$ values can be included in Eq. (8) in order to estimate the space charge tune shift of the bunch eigenfrequencies for a given bunch length. The chain line demonstrates again the difference between $Q_{s^{*}}$ which describes the tune shift and the longitudinal dipole frequency.

\section{Transverse decoherence}

\section{Linear decoherence}

First, we discuss the linear transverse decoherence due to chromaticity, i.e., the only source of the tune shift is the

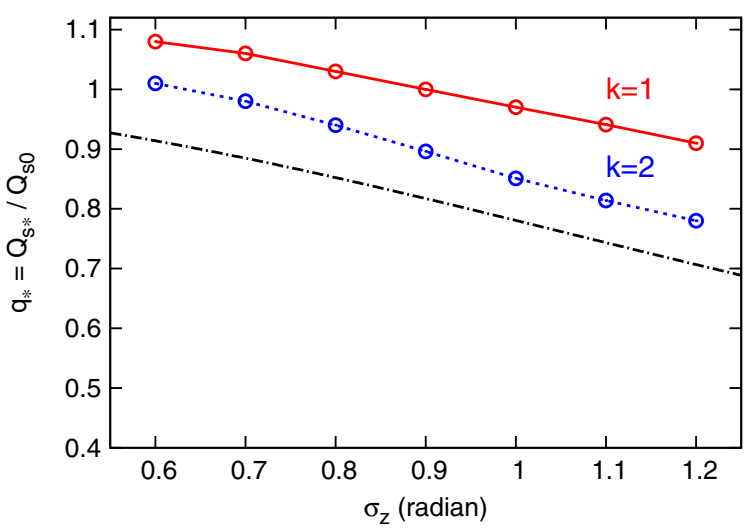

FIG. 5. Summary of the simulation scans for the effect of the bunch length on the eigenfrequencies of the head-tail modes $k=$ 1 and $k=2$ with space charge. For comparison, the chain curve shows the longitudinal dipole frequency from Eq. (4) for a Gaussian bunch. linear dependence of the betatron tune shift on the momentum shift $\Delta Q_{\xi} / Q=\xi \Delta p / p$. As a result of an initial transverse displacement $\bar{x}(\tau)=A_{0}$, a bunch oscillates in the corresponding plane (here $x$ ). As we consider the linear case, all the particles have the identical synchrotron frequency $Q_{s} f_{0}$. The betatron phase shift related to the bare tune $Q_{0}$ has a harmonic dependency along a synchrotron period. Hence, after one synchrotron oscillation, the betatron phase shift is exactly compensated and the transverse amplitude is equal to the initial displacement $A_{0}$. Assuming the Gaussian momentum distribution, the amplitude of the bunch offset evolves with the turn number $N$ as [12]

$$
A(N)=A_{0} \exp \left\{-2\left(\frac{\xi Q_{0} \delta_{p}}{Q_{s}} \sin \left(\pi Q_{s} N\right)\right)^{2}\right\}
$$

here $\delta_{p}$ is the normalized rms momentum spread. Figure 6 shows an example for bunch decoherence after a rigid kick. It demonstrates that a higher chromaticity provides a faster decoherence, and that after the synchrotron period $N_{s}=$ $1 / Q_{s}$ the initial offset amplitude appears again, which is called recoherence.

\section{Decoherence with space charge}

Transverse space charge causes a betatron frequency shift, which depends on the particle transverse amplitude and on the longitudinal particle position in the bunch. The decoherence behavior is thus very different from the linear decoherence at low bunch intensities Eq. (9). Figure 7 shows examples of the bunch oscillations after a rigid kick for three different values of the space charge parameter. The chromaticity corresponds to $\chi_{b}=4.5$, where $\chi_{b}=Q_{0} \xi L_{b} /(\eta R)$ is the chromatic phase shift over the full bunch length $L_{b}$; the bunch rms length is $\sigma_{z}=$ $1.06 \mathrm{rad}$. We observe the periodic recoherence with the periodicity 770 turns $(q=7$, top), 1270 turns $(q=12$,

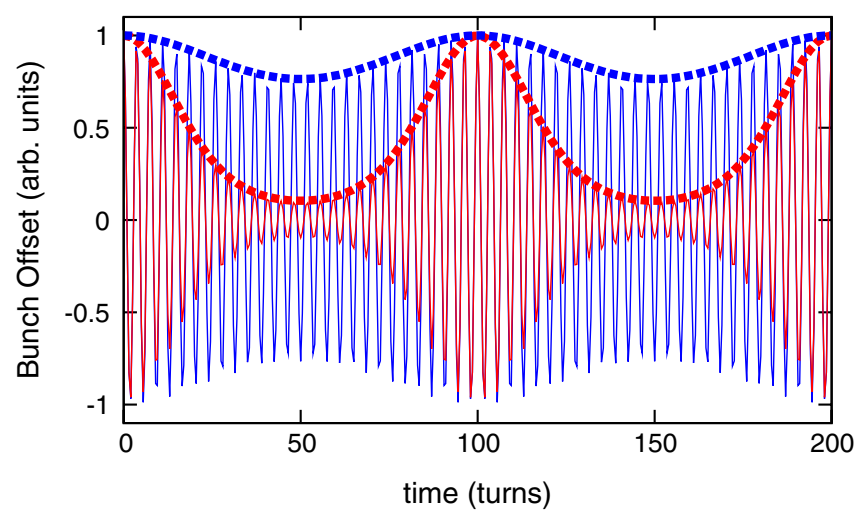

FIG. 6. A particle tracking simulation for a Gaussian bunch after an offset kick $\bar{x}(\tau)=$ const without space charge and for a linear rf bucket, $Q_{s}=Q_{s 0}=0.01$. The full lines show the time evolution of the bunch offset for the chromaticities $\xi Q_{0}=-4.3$ (blue) and $\xi Q_{0}=-12.5$ (red), the dashed lines are analytical results and are given by Eq. (9). 


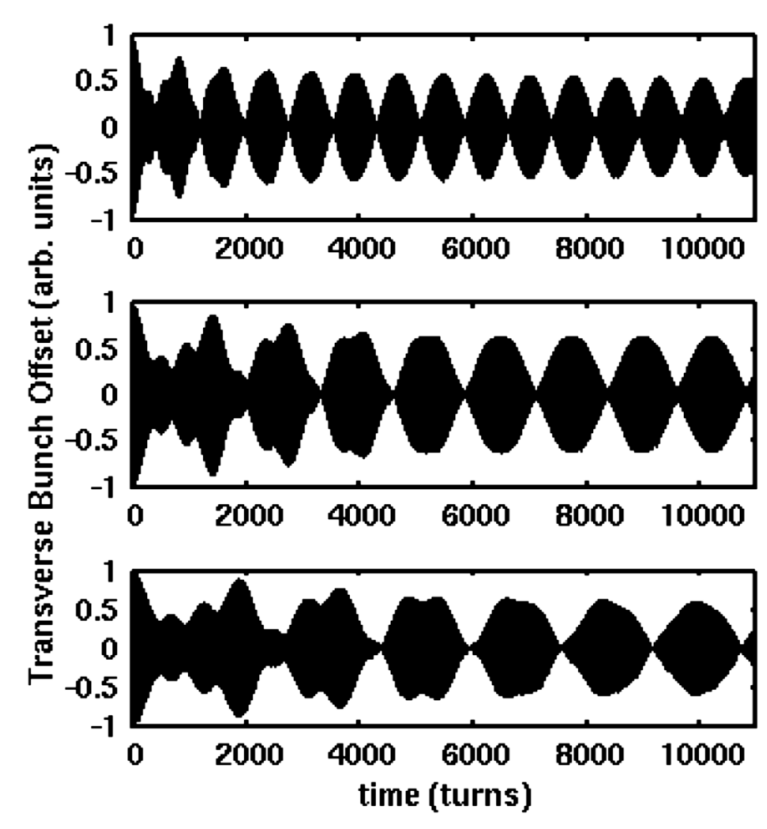

FIG. 7. Transverse bunch decoherence from particle tracking simulations for a Gaussian bunch after a rigid kick $\bar{x}(\tau)=$ const for different space charge parameters. Top plot: $q=7$, middle plot: $q=12$, and for the bottom plot: $q=16$. The bare synchrotron tune is $Q_{s 0}=0.01$, i.e., the low-intensity recoherence has a periodicity of 100 turns. After the transition period of higher-order mode damping, the periodicity always corresponds to the frequency difference $\Delta Q=Q_{k=1}-Q_{k=0}$. Top plot: $\Delta Q_{k=1}=0.13 Q_{s 0} \quad$ (periodicity 770 turns), middle plot: $\Delta Q_{k=1}=0.079 Q_{s 0}$ (periodicity 1270 turns), and for the bottom plot $\Delta Q_{k=1}=0.061 Q_{s 0}$ (periodicity 1640 turns).

middle), and 1640 turns $(q=16$, bottom), while the lowintensity recoherence would have a periodicity of 100 turns for the same parameters.

The key in understanding the decoherence for a bunch with transverse space charge is the representation of the initial kick as a superposition of the bunch head-tail eigenmodes,

$$
A_{0}=\sum_{k} a_{k} \exp \left(-i \frac{\chi_{b} \tau}{\tau_{b}}+i \phi_{k}\right) \bar{x}_{k}(\tau)
$$

where we have extracted the chromatic phase shift along the bunch with the corresponding phase $\phi_{k}$ for each eigenfunction. The second key is the fact that the different eigenmodes are prone to Landau damping mechanisms, but with different intensity thresholds and damping rates. Landau damping due to the space charge tune spread along the bunch $[11,13,14]$ is the most important mechanism in the beam parameter regime considered in the simulations of this work. In the presence of space charge especially the negative and the high- $k$ eigenmodes present in the initial kick Eq. (10) are quickly suppressed, so that after a transition period a mixture of the survived eigenmodes continues to oscillate.
In Ref. [15] we have discussed in detail the case $q=1$, where all the head-tail modes $k \geq 1$ are strongly suppressed by Landau damping such that the mode $k=0$ is left alone. For stronger space charge, as in Fig. 7, the modes $k \geq 2$ are damped and the resulting oscillation is the mixture of the $k=0$ and $k=1$ modes. The recoherence periodicity seen in Fig. 7 corresponds exactly to the frequency difference between these two modes, as it is the case for the wave beating. In a real machine there are often nonlinear damping mechanisms which would further suppress the $k=0$ and $k=1$ modes, but in the simulation we only have the space charge induced Landau damping which is zero for the $k=0$ mode and is very weak for the $k=1$ mode at these $q$ parameters.

It is obvious, and can be seen in Eq. (10), that the composition of the eigenmodes after a rigid kick depends on the chromaticity. This is also demonstrated in Fig. 8 which shows a comparison of the bunch decoherence for three different chromaticities. The bunch parameters correspond to Fig. 7; the space charge parameter is chosen as $q=7$. We see that the periodicity of 770 turns does not change. It corresponds to the frequency difference $\Delta Q=$ $Q_{k=1}-Q_{k=0}=0.13 Q_{s 0}$. The reason for the different oscillation amplitudes in Fig. 7 is the increasing contribution of higher-order modes $k \geq 2$ with growing $\xi$ in the eigenmode mixture of the initial rigid bunch offset [see Eq. (10) ]. Recall that these modes are quickly suppressed for the parameters of the bunch and the resulting recoherence is a beating of the remaining $k=0$ and $k=1$ modes.

The airbag [2] eigenmodes $\bar{x}_{k}(\tau)=A \cos \left(k \pi \tau / \tau_{b}\right)$ can be taken as a reasonable approximation [14] of the eigenfunctions in a Gaussian bunch. The rigid offset decomposition Eq. (10) can then be solved and the resulting mode coefficients are $a_{0}=2 / \chi_{b} \sin \left(\chi_{b} / 2\right)$,

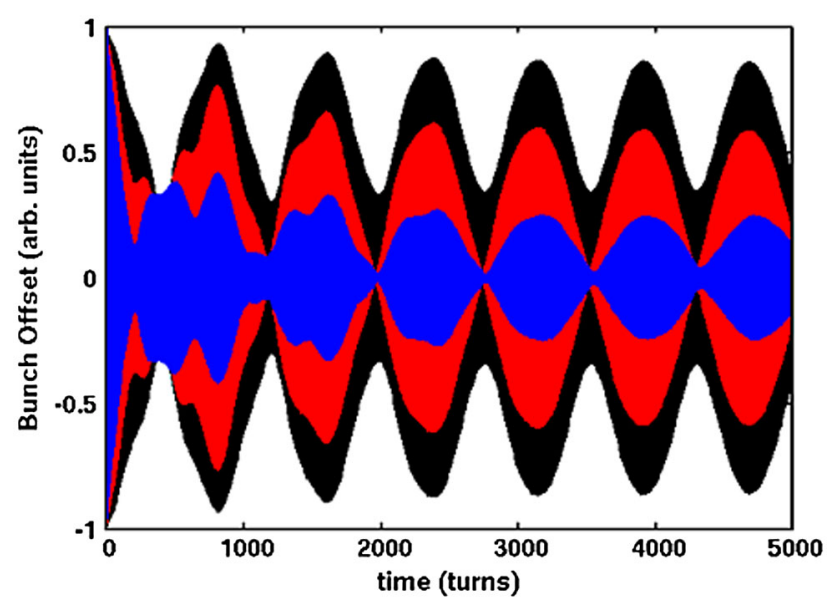

FIG. 8. Transverse bunch decoherence for a bunch with space charge parameter $q=7$ from particle tracking simulation for different chromaticities. The black curve: $\chi_{b}=3$, the red curve: $\chi_{b}=4.5$, and for the blue curve: $\chi_{b}=6$. The recoherence results from a mixture of the $k=0$ mode and $k=1$ mode, $\Delta Q_{k=1}=0.13 Q_{s 0}$ (periodicity 770 turns). 


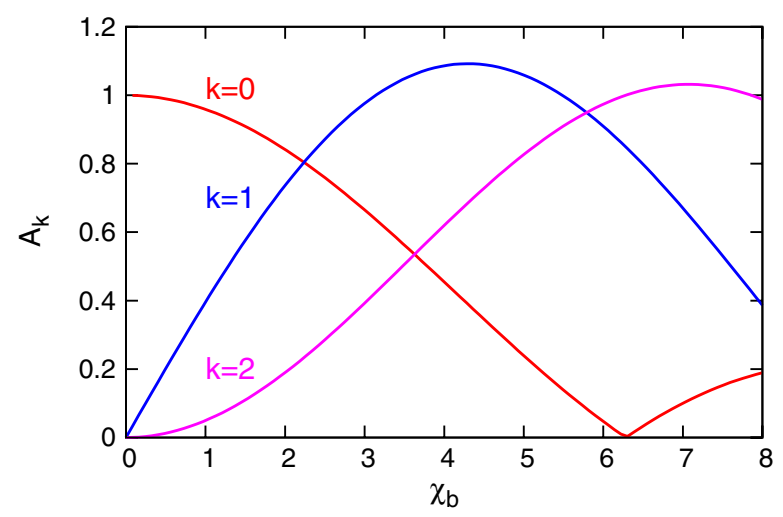

FIG. 9. Relative amplitudes of the airbag bunch eigenmodes for the rigid offset $\bar{x}(\tau)=$ const as a function of the chromatic phase shift $\chi_{b}=Q_{0} \xi L_{b} /(\eta R)$.

$a_{1}=4 \chi_{b} /\left|\chi_{b}^{2}-\pi^{2}\right| \cos \left(\chi_{b} / 2\right), \quad$ and $\quad a_{2}=4 \chi_{b} / \mid \chi_{b}^{2}-$ $4 \pi^{2} \mid \sin \left(\chi_{b} / 2\right)$. The negative modes have the same coefficients but can be disregarded in the case of a bunch with space charge [11,14], because of their large damping rates. These coefficients are plotted in Fig. 9, where we see that for the chromaticity range of interest the relative part of the $k=2$ mode increases with growing $\chi_{b}$. The higher-order modes follow this trend. The contribution of the $k=0$ and $k=1$ modes thus decreases as we also can observe in the simulations, see Fig. 8. A perfect agreement with the coefficients in Fig. 9 cannot be expected, since the analytical model is for an airbag [2] bunch.

\section{MEASUREMENTS}

Transverse decoherence experiments have been performed in the heavy-ion synchrotron SIS18 [16] at GSI Darmstadt. Bunches of $\mathrm{Ar}_{40}^{18+}$ ions were stored at the energy of $100 \mathrm{MeV} / \mathrm{u}$ and kicked transversally with a kick duration of one turn. The rf harmonic number was $h=4$ and all the four bunches had generally an identical behavior. The beam position monitors (BPMs) provide a higher quality signal in the vertical plane than in the horizontal one due to a smaller plate gap, thus we use the vertical BPM signals in the results presented here. The vertical bare tune was around $Q_{0}=4.31$ although it could vary for different intensities and machine parameters. SIS18 general parameters are $R=34.492 \mathrm{~m}, \gamma_{t}=5.45$, and $\xi \approx-1.4$.

Similar to the theory section, first we discuss the longitudinal coherent dipole frequency. Figure 10 demonstrates the bunch spectrum obtained from the sum BPM signal. The satellites of the central frequency are well resolved; the peaks are equidistant which provides the longitudinal dipole frequency. The longitudinal dipole frequency determined in this way is $Q_{\text {dip }}=2.5 \times 10^{-3}$; the peak rf voltage was $V_{0}=9 \mathrm{kV}$ here. The bare synchrotron tune can also be accurately determined using Eq. (5) and it is $Q_{s 0}=$ $3.24 \times 10^{-3}$ in this case. Note the large difference between

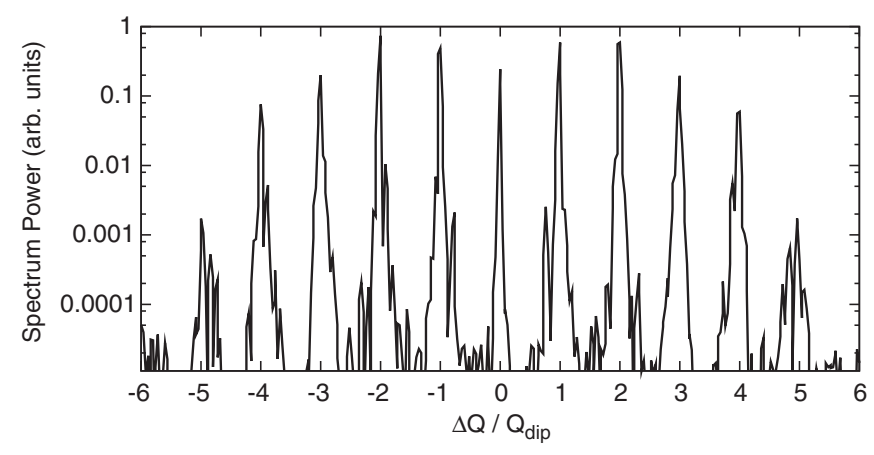

FIG. 10. An example for a longitudinal coherent spectrum from BPM bunch measurements at SIS18. The spectrum corresponds to the 22nd harmonic at the frequency of $13.073 \mathrm{MHz}$, $\Delta Q=\left(f-n f_{0}\right) / f_{0}$.

the bare synchrotron frequency and the dipole frequency. Using the curves from Fig. 1 we can obtain the rms bunch length $\sigma_{z}=1.0 \mathrm{rad}$, which is a typical length in the experiments at SIS18.

The first example for the decoherence measurements is presented in Fig. 11 and 12. Figure 11 shows the turnper-turn transverse bunch offset after the kick. Figure 12 demonstrates the spectrum of these bunch oscillations; the frequency on the horizontal axis is normalized by the bare synchrotron tune. The red line is for the spectrum of the whole bunch and shows mainly peaks of two modes which we can identify as the $k=0$ mode and the $k=2$ mode. If we calculate a Fourier transform for the bunch head, its spectrum (the blue line) clearly reveals other peaks, so that we can identify five head-tail modes, see Fig. 12. The spectrum is very different from the case without collective effects: the lines are not equidistant, the negative modes $(k<0)$ are suppressed. The fact that the mode tune shifts are consistent with the space charge model can be proved by calculating the space charge parameter,

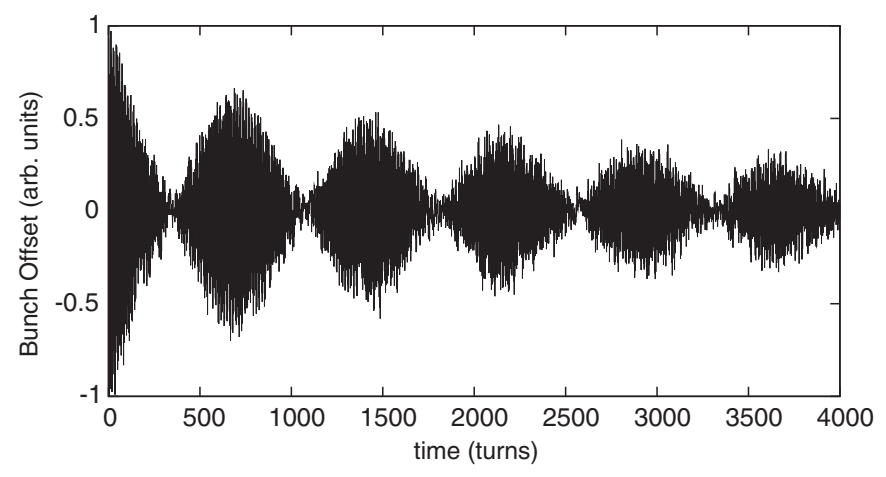

FIG. 11. Time evolution of the bunch offset in the vertical plane at SIS18 after a transverse kick. The recoherence periodicity corresponds to the mix of the dominating head-tail modes $k=0$ and $k=2$ with $\Delta Q_{k=2}=1.35 \times 10^{-3}$, giving the periodicity of 740 turns. 


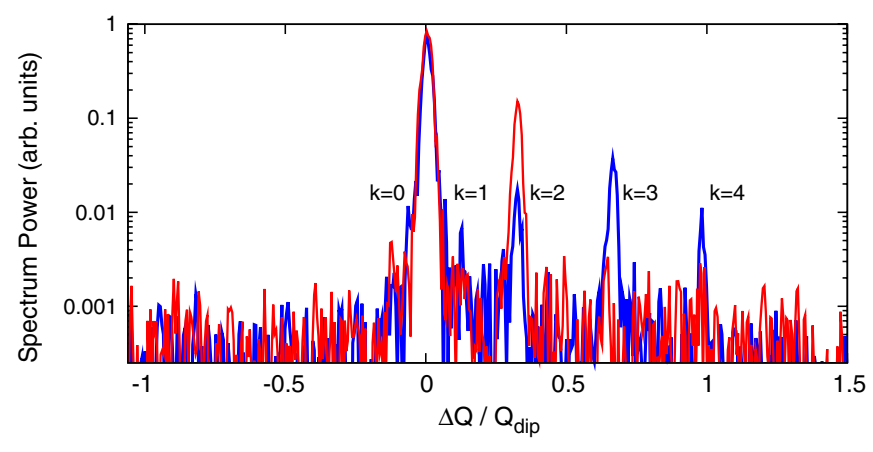

FIG. 12. Transverse coherent spectrum for the bunch from Fig. $11, Q_{s 0}=4.04 \times 10^{-3}$. The red spectrum is obtained from a frequency analysis of the complete bunch offset, while the blue spectrum is a result of a frequency analysis for the bunch head. In the complete bunch spectrum the mode $k=2$ dominates, and the bunch head spectrum reveals the uneven modes $k=1, k=3$ but also the mode $k=4$. The frequencies of the head-tail modes provide the space charge parameter $q \approx$ 10, see blue circles in Fig. 13. The bunch length corresponds to $\sigma_{z}=0.66 \mathrm{rad}, \chi_{b} \approx 3$.

$$
q=\frac{k^{2} q_{*}^{2}-\left(\Delta Q_{k} / Q_{s 0}\right)^{2}}{\Delta Q_{k} / Q_{s 0}}
$$

which corresponds to the model Eq. (8). The synchrotron oscillation parameter $q_{*}$ for the modes $k=1$ and $k=2$ is obtained from the results given in Fig. 5. $\Delta Q_{k}$ is the tune shift of the bunch mode from the measured spectrum. Here and for the examples to follow we summarize the space charge parameters $q$ obtained from the different eigenfrequencies of the spectra in Fig. 13. The relevant bunch parameters are summarized in Table I. The values for the modes from Fig. 12 are shown in Fig. 13 with the blue circles, $q \approx 10$. Since this was a rather short bunch, $\sigma_{z}=0.66 \mathrm{rad}$, the $q_{*}$

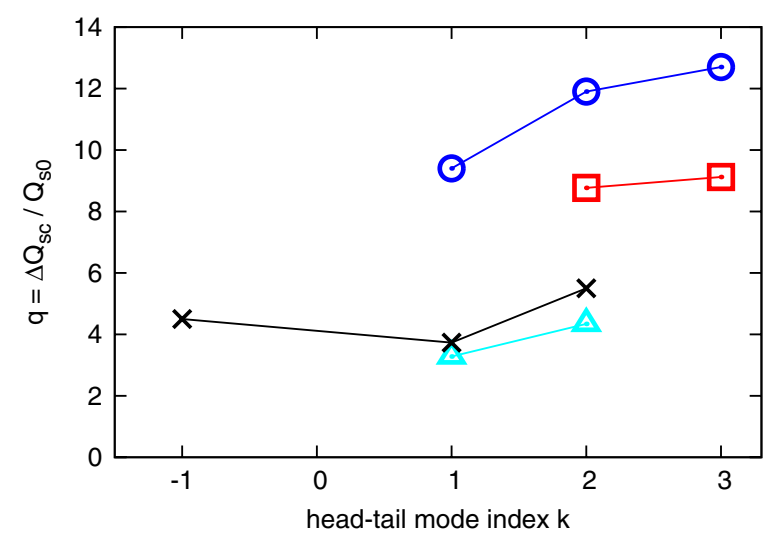

FIG. 13. Summary for the space charge parameter determined from the coherent head-tail spectra of different $\mathrm{Ar}_{40}^{18+}$ bunched beams in the SIS18 synchrotron. The method is given by Eq. (11) , with the coefficients $q_{*}$ corresponding to Fig. 5. The spectra are shown in Fig. 12 (blue circles), Fig. 15 (red squares), Fig. 18 (black crosses), and in Fig. 20 (cyan triangles).
TABLE I. Bunch parameters for the signals shown in this paper and the space charge parameter $q$ obtained from the transverse spectra. The $q$ values from the different head-tail modes for every bunch are summarized in Fig. 13. In the first case the rf voltage was $V_{0}=14 \mathrm{kV}$; in the last three cases $V_{0}=9 \mathrm{kV}$.

\begin{tabular}{lcccccc}
\hline \hline Signals & $\begin{array}{c}\text { Symbols in } \\
\text { Fig. 13 }\end{array}$ & $\begin{array}{c}\sigma_{z}, \\
\text { rad }\end{array}$ & $\chi_{b}$ & $\begin{array}{c}Q_{\text {dip }}, \\
10^{-3}\end{array}$ & $\begin{array}{c}Q_{s 0}, \\
10^{-3}\end{array}$ & $q$ \\
\hline Figs. 11 and 12 & Blue circles & 0.66 & $\approx 3$ & 3.63 & 4.0 & $\approx 10$ \\
Figs. 14 and 15 & Red squares & 1.15 & $\approx 5$ & 2.35 & 3.24 & $\approx 9$ \\
Figs. 17 and 18 & Black crosses & 1.2 & $\approx 5$ & 2.28 & 3.24 & $\approx 4.5$ \\
Figs. 19 and 20 & Cyan triangles & 1.0 & $\approx 0$ & 2.5 & 3.24 & $\approx 4$ \\
\hline \hline
\end{tabular}

parameter was close to 1.0 and thus it was possible to estimate the space charge parameter for the $k=3$ mode as well.

Figure 13 demonstrates a certain consistency between different head-tail modes for the space charge parameter, that, however, cannot be expected to be perfect. The model Eq. (8) is based on the airbag [2] bunch which is reasonable, but still an approximation, for a Gaussian bunch [14]. The bunch spectra are also weakly affected by the facility impedances, image charges, and nonlinear field components neglected in our analysis. Finally, in our simulations Gaussian bunch profiles in the transverse and in the longitudinal plane have been assumed. It is a good, but not exact, description for the bunches in the machine experiments.

The space charge parameter $q=\Delta Q_{\mathrm{sc}} / Q_{s 0}$ can be additionally estimated using Eq. (1) and the measured bunch parameters. The particle number and the bunch length could be measured with a reasonable accuracy. The transverse beam radius, which enters the space charge tune shift as squared ( $\varepsilon_{y}=a_{y}^{2} Q_{0 y} / R, a_{y}$ is the vertical rms radius) and is thus especially important, could not be determined with a satisfactory precision, as it was also the case in the previous coasting-beam measurements [17] at SIS18.

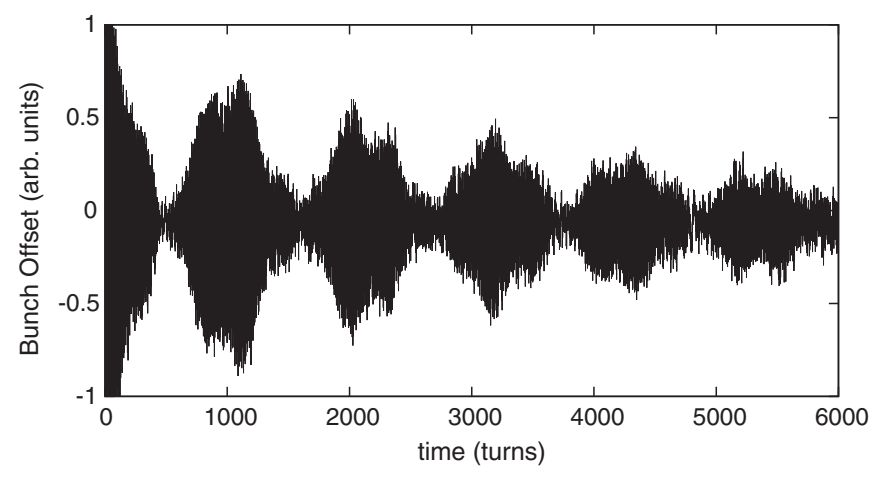

FIG. 14. Time evolution of the bunch offset in the vertical plane at SIS18 after a transverse kick. The recoherence periodicity corresponds to the mix of the dominating head-tail modes $k=0$ and $k=2$ with $\Delta Q_{k=2}=0.91 \times 10^{-3}$, giving the periodicity of 1100 turns. 


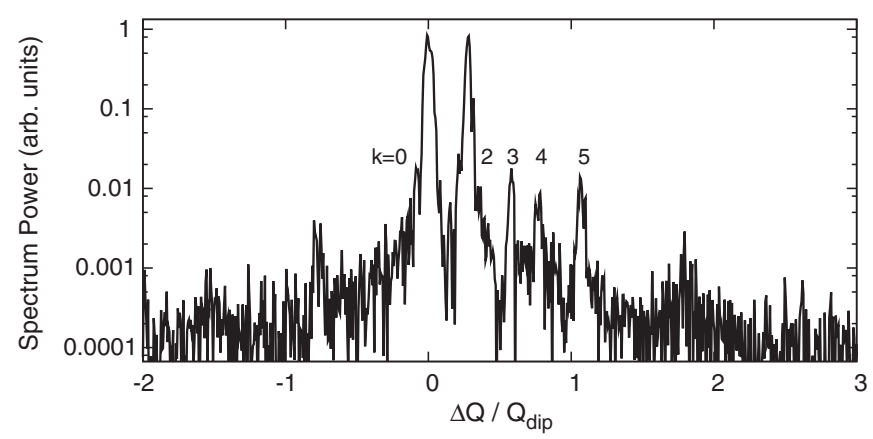

FIG. 15. Transverse coherent spectrum for the bunch from Fig. $14, Q_{s 0}=3.24 \times 10^{-3}$. Head-tail modes up to $k=5$ are well seen, except for the modes $k=1$. The frequencies of the head-tail modes provide the space charge parameter $q \approx 9$, see red squares in Fig. 13. The bunch length corresponds to $\sigma_{z}=$ $1.15 \mathrm{rad}, \chi_{b} \approx 5$.

As an example, here we provide an estimation for the bunch presented in Figs. 11 and 12. The transverse rms emittances were $\epsilon_{y}=6.2 \mathrm{~mm} \mathrm{mrad}, \epsilon_{x}=8.4 \mathrm{~mm} \mathrm{rad}$, number of ions per bunch was $5.1 \times 10^{9}$. Using these parameters, the bunch length, and the bare synchrotron tune (see Table I), we obtain from Eqs. (1) and (2) $q_{\text {est }} \approx 7$. In this work we make no claim on a perfect agreement of the $q$ values obtained from the transverse spectra with the $q$ estimations provided by the bunch parameters and Eq. (1), mainly due to the uncertainty in the transverse beam size measurements at SIS18.

In the next example we show a longer bunch, $\sigma_{z}=$ $1.15 \mathrm{rad}$, due to a lower rf voltage, see Table I. The transverse bunch oscillations after the kick are shown in Fig. 14 and the corresponding spectrum is shown in Fig. 15. In comparison to the previous example (Figs. 11 and 12), the bunch here is longer, but the particle number is higher and the synchrotron tune is larger, thus the space charge parameter is similar, $q \approx 9$. As we can see in Fig. 15, the

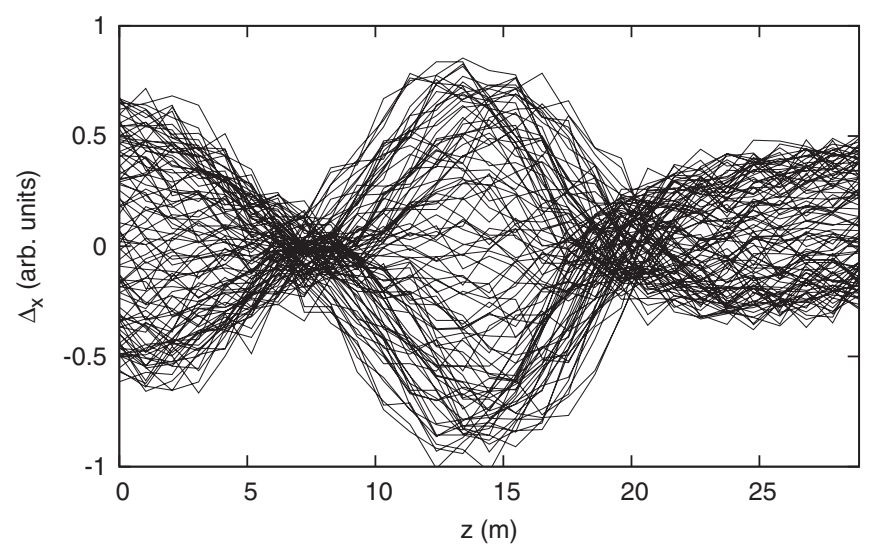

FIG. 16. Traces of the transverse bunch signal for 100 consecutive turns for the bunch from Figs. 14 and 15. This result proves that the mode $k=2$ dominates during the process of bunch decoherence.

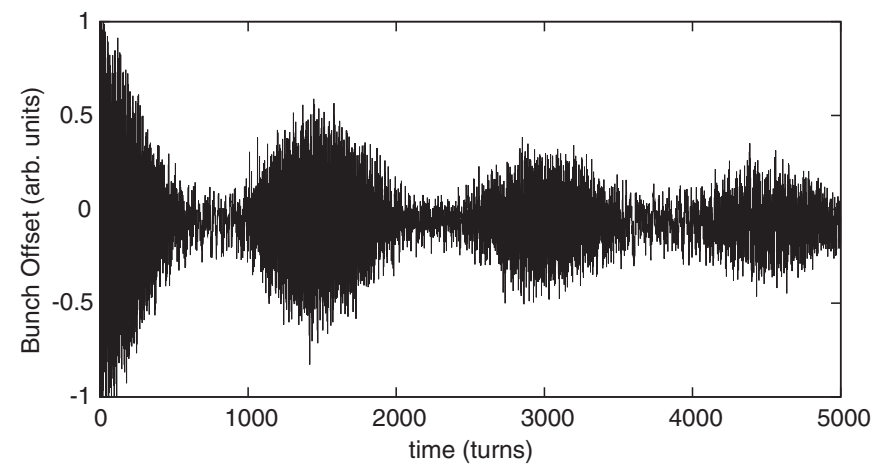

FIG. 17. Time evolution of the bunch offset in the vertical plane at SIS18 after a transverse kick. The recoherence periodicity corresponds to the mix of the dominating head-tail modes $k=0$ and $k=1$ with $\Delta Q_{k=1}=0.68 \times 10^{-3}$, giving the periodicity of 1470 turns.

spectrum is dominated by two modes, the $k=0$ mode at the bare tune, and another one at $\Delta Q=0.91 \times 10^{-3}$, which gives the periodicity of the bunch recoherence, see Fig. 14. The mode $k=1$ is suppressed as is the case in the previous example, and it is to be expected that here we have the $k=2$ mode again. Additionally, this could be proved as follows. Plotting the bunch vertical traces and subtracting the total bunch offset, thus reducing the contribution of the $k=0$ mode, we observe a clear two-knot structure of the $k=2$ modes, see Fig. 16. The frequencies of the further peaks in Fig. 15 correspond rather well to the space charge model with $q \approx 9$.

In the next example we demonstrate a bunch decoherence dominated by a mixture of the $k=0$ mode with the $k=1$ mode; the bunch oscillations are shown in Fig. 17, the spectrum is shown in Fig. 18. The horizontal chromaticity was partly compensated, by a half of the natural value, the associated nonlinearities probably contributed to establishing of the longer bunch and to a

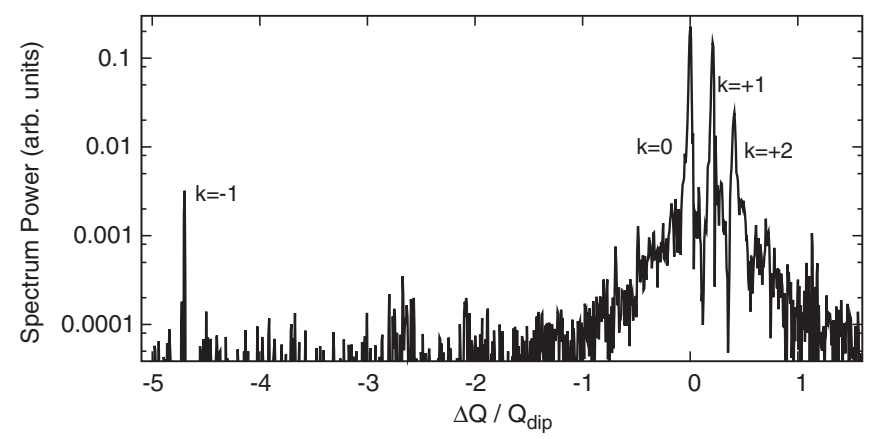

FIG. 18. Transverse coherent spectrum for the bunch from Fig. 17, $Q_{s 0}=3.24 \times 10^{-3}$. The mode $k=1$ dominates, the spectrum shows clearly the mode $k=-1$, with the eigenfrequency corresponding well to the model Eq. (8). The frequencies of the head-tail modes provide the space charge parameter $q \approx 4.5$, see black crosses in Fig. 13. The bunch length corresponds to $\sigma_{z}=1.2 \mathrm{rad}, \chi_{b} \approx 5$. 
stronger damping of the $k=2$ mode. The recoherence is thus quite slower, nearly one and a half thousand turns (see Fig. 17), which is given by the frequency of the $k=1$ mode in good agreement with the bunch spectrum, Fig. 18. Another outstanding feature of this spectrum is the clear presence of the $k=-1$ mode, with the frequency shifted strongly downwards, in a good quantitative agreement with the space charge model, see black crosses in Fig. 13. The strong negative shifts for the $k<0$ modes have been predicted by the space charge models [2,11] and observed in the numerical simulations [3,9]. For strong space charge, due to nearness of the negative head-tail modes to the incoherent tune these modes are strongly Landau damped. In part, the presence of the $k=-1$ mode was probably possible here due to rather moderate space charge $q \approx 4.5$ in this case.

The transverse decoherence observed in the case presented in Figs. 19 and 20, is very different from the third example, Fig. 17, although the space charge parameter is similar, $q \approx 4$, as well as the bunch length, see Table I. We see that the recoherence periodicity is quite faster which is due to the dominance of the $k=2$ mode as it is confirmed in the bunch spectrum, see the red line in Fig. 20. More remarkable, the bunch decoherence in Fig. 19 shows a much weaker amplitude drop between the recoherence peaks. The reason is the compensated vertical chromaticity to nearly zero, according to the set parameters. This is predicted by the linear theory Eq. (9), also shown in Fig. 6. According to the interpretation of the mode mixture, at a small chromaticity the part of the $k=0$ mode is very large, see Fig. 9. The spectrum from the measurements in Fig. 20 confirms this. The relatively small part of the $k=2$ mode provides the periodicity of a weak recoherence. For the determination of the space charge parameter, the eigenfrequency of the $k=1$ mode is needed which could be obtained by a frequency analysis of the bunch head

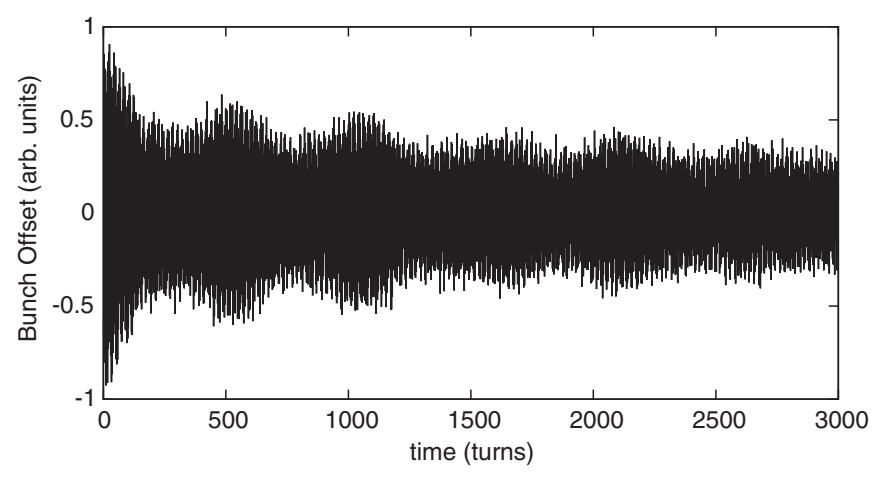

FIG. 19. Time evolution of the bunch offset in the vertical plane at SIS18 after a transverse kick. The vertical chromaticity was compensated for this beam to $\xi_{y} \approx 0$. The recoherence periodicity corresponds to the mix of the dominating head-tail modes $k=0$ and $k=2$ with $\Delta Q_{k=2}=1.86 \times 10^{-3}$, giving the periodicity of 540 turns.

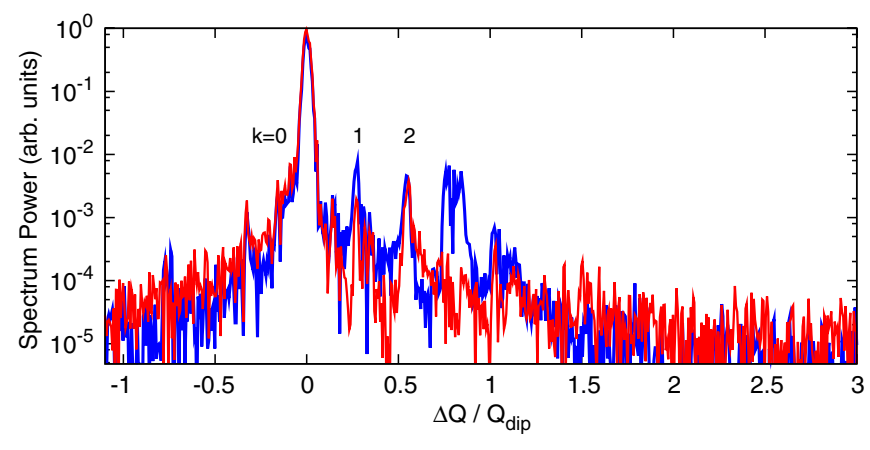

FIG. 20. Transverse coherent spectrum for the bunch from Fig. $19, Q_{s 0}=3.24 \times 10^{-3}$. The red spectrum is obtained from a frequency analysis of the complete bunch offset, while the blue spectrum is a result of a frequency analysis for the bunch head. The mode $k=0$ highly dominates, in the complete bunch spectrum the mode $k=2$ is stronger, and the bunch head spectrum reveals the uneven modes $k=1, k=3$. The frequencies of the head-tail modes provide the space charge parameter $q \approx 4$, see cyan triangles in Fig. 13. The bunch length corresponds to $\sigma_{z}=1.0 \mathrm{rad}, \chi_{b} \approx 0$.

oscillations, see the blue line in Fig. 20, and the resulting $q$ values in Fig. 13 (cyan triangles).

\section{CONCLUSIONS}

The transverse decoherence and coherent eigenspectra in long bunches with space charge have been studied using measurements at the SIS18 heavy-ion synchrotron and particle tracking simulations.

A model Eq. (8) for the combined effect of space charge and nonlinear synchrotron oscillations has been formulated, with the fitting parameter $q_{*}$ obtained from the particle tracking simulations for the low-order head-tail modes. The space charge parameter $q=\Delta Q_{\mathrm{sc}} / Q_{s 0}$ of the bunch can be determined for every head-tail mode from the corresponding frequency shift $\Delta Q_{k}$, see Eq. (11), according to the given bunch length.

The transverse decoherence in bunches with space charge has been observed experimentally and quantitatively explained, using simulations and analytic models. An initial rigid bunch offset can be decomposed into headtail modes. The chromaticity determines the contribution of the different head-tail modes. Using the airbag [2] eigenmodes as an approximation for the bunch head-tail modes, the relative amplitudes can be found analytically, see Fig. 8.

Different head-tail modes experience also different Landau damping rates. After a transition period the bunch oscillation is a combination of the remaining modes. For example, it can be a mix of the $k=0$ mode and $k=1$ mode. The periodicity of the bunch recoherence corresponds then to the frequency difference between these two modes. Our simulation examples demonstrate this explanation of the bunch decoherence for different space 
charge parameters and for different chromaticities, see Figs. 7 and 8. An important Landau damping mechanism in bunches with space charge is due to the variation of the space charge tune shift along the bunch [11,13,14].

Experimental observations of the transverse bunch decoherence with space charge in the SIS18 heavy-ion synchrotron at GSI are presented. The space charge parameter $q$ has been determined from the bunch spectra for different head-tail modes, summarized in Fig. 13. The work is focused on the frequency positions of the bunch spectrum peaks; the peak widths in our observations were dominated by the Fourier window so the physical peak width is out of the work scope. With increasing bunch length we observe that nonlinear synchrotron oscillations modify the headtail mode frequencies. The bunch decoherence always corresponded to the mix of the dominating modes, in our case the $k=0$ and $k=1$ modes or the $k=0$ and $k=2$ modes. Compared to the simulation it is more difficult to predict which modes would be faster suppressed due to additional damping mechanisms in a real machine. In the experiment the oscillations are further damped after the transition period, possibly due to the nonlinear magnet field errors. The periodicity of the recoherence was exactly confirmed by the mode frequencies from the spectra. A direct comparison of the first two examples (Figs. 11 and 12 vs Figs. 14 and 15) demonstrates the role of the bunch length. A comparison of the fourth example (Figs. 19 and 20) with the others demonstrates the role of the chromaticity: at a nearly zero chromaticity the mode $k=0$ dominates the bunch decoherence alone. The third example (Figs. 17 and 18) shows a decoherence with a pronounced flat between the recoherence peaks, corresponding to the mix of the $k=0$ and $k=1$ modes.

The results of this work apply to the evolution of a possible transverse injection offset during bunch-to-bucket transfer from one ring to another. Transverse coherent spectra can be used not only to measure the betatron tune, the head-tail mode frequencies can be used to extract useful information about the long intense bunches, for example the incoherent space charge tune shift. The effects of space charge and rf nonlinearity have also direct consequences for the chromaticity measurements which use the decoherence/recoherence after a kick [18]. As we have previously discussed in Ref. [15], Landau damping due to space charge suppresses the $k \geq 1$ modes with different damping rates and makes the head-tail phase shift within the bunch inapplicable for a chromaticity measurement. Only in the case when the eigenmode $k=0$ continues to oscillate alone is it possible to extract the chromaticity using a different (from the no-space-charge case [18]) expression. However, in a general case a combination of the eigenmodes survives during the first recoherence periods, and the methods from $[15,18]$ do not provide a useful chromaticity calculation, as we have also observed for the signals presented in this paper. Hence, the decoherence with space charge and rf nonlinearity should be analyzed in detail in future papers.

[1] R. Jones and H. Schmickler, CERN Accelerator School, Report No. CERN-2006-002, 2003, p. 75.

[2] M. Blaskiewicz, Phys. Rev. ST Accel. Beams 1, 044201 (1998).

[3] O. Boine-Frankenheim and V. Kornilov, Phys. Rev. ST Accel. Beams 12, 114201 (2009).

[4] A. Hofmann and F. Pedersen, IEEE Trans. Nucl. Sci. 26, 3526 (1979).

[5] S. S. Nagaitsev, M. J. Ellison, T. J. Elisson, M. S. Ball, V. Derenchuk, G. East, B. J. Hamilton, and P. Schwandt, Part. Accel. 56, 181 (1997).

[6] O. Boine-Frankenheim, T. Shukla, Phys. Rev. ST Accel. Beams 8, 034201 (2005).

[7] O. Boine-Frankenheim and V. Kornilov, in Proceedings of ICAP2006, Chamonix Mont-Blanc (2006).

[8] G. Rumolo and F. Zimmermann, Phys. Rev. ST Accel. Beams 5, 121002 (2002).

[9] V. Kornilov and O. Boine-Frankenheim, in Proceedings of the 10th International Computational Accelerator Physics Conference ICAP2009, San Francisco (2009).

[10] A. Burov and V. Lebedev, Phys. Rev. ST Accel. Beams 12, 034201 (2009).

[11] A. Burov, Phys. Rev. ST Accel. Beams 12, 044202 (2009); 12, 109901(E) (2009).

[12] R. E. Meller, A. W. Chao, J. M. Peterson, S. G. Peggs, M. Furman, SSC Report No. SSC-N-360, 1987.

[13] V. Balbekov, Phys. Rev. ST Accel. Beams 12, 124402 (2009).

[14] V. Kornilov and O. Boine-Frankenheim, Phys. Rev. ST Accel. Beams 13, 114201 (2010).

[15] V. Kornilov, O. Boine-Frankenheim, Proceedings of the 46th ICFA Advanced Beam Dynamics Workshop HB2010, Morschach, Switzerland (2010), p. 101.

[16] K. Blasche and B. Franczak, in Proceedings of EPAC 1992, Berlin, Germany (1992), p. 9.

[17] S. Paret, V. Kornilov, O. Boine-Frankenheim, and T. Weiland, Phys. Rev. ST Accel. Beams 13, 022802 (2010).

[18] D. Cocq, O. R. Jones, and H. Schmickler, in Proceedings of BIW98, Stanford, CA, USA (1998) 